\title{
Expectativas ciudadanas frente a una asamblea constituyente: La experiencia chilena
}

\author{
Citizen Expectations of a Constituent Assembly: \\ The Chilean Experience
}

\author{
María Cristina Escudero ${ }^{1}$
}

Cómo citar este artículo: Escudero, M.C. (2021). Expectativas ciudadanas frente a una asamblea constituyente: La experiencia chilena. Revista de Ciencias Sociales Ambos Mundos, (2), 79-89. https://doi.org/10.14198/ambos.21034

\begin{abstract}
Resumen
Las expectativas que tiene Chile respecto al desempeño y ejercicio del poder constituyente de la Convención Constitucional que se inició el 4 de julio de 2021, no se diferencia de lo que parte de la experiencia comparada en Latinoamérica a esperado de sus respectivos procesos constituyentes de finales del siglo XX y comienzos del siglo XXI. En los hechos, desde que se empieza a consolidar la demanda por una nueva constitución, la Asamblea Constituyente es la opción que refleja una instancia que se aleja de la política de los partidos, participativa y reflejo de la voluntad del pueblo o ciudadana. La elección de convencionales constituyentes de mayo de 2021, es el resultado de esta convicción que ya se había sincerado en el proceso participativo para la elaboración de las bases ciudadanas para una nueva constitución, impulsada por el gobierno de Bachelet en 2016.
\end{abstract}

Palabras claves: Chile; Proceso Constituyente; Convención Constitucional; Asamblea Constituyente; Encuentros Locales; Autoconvocados.

Abstract

Chile's expectations regarding the performance and exercise of the constituent power of the Constitutional Convention, that began its work on 4 July 2021, is no different from the expectation of previous Latin American constitution-making experiences. Since the demand for a new constitution began to consolidate, the Constituent Assembly represents the participatory option that reflects a move away from party politics and closer to the people's will. The election of Constituent Assembly members in May 2021 is the result of this expectation expressed previously. In that realm, a relevant antecedent was President Bachelet participatory process to build citizen bases for a new constitution in 2016.

Keywords: Chile; Constitution-Making Process; Constitutional Convention; Constituent Assembly; Local Self-Convened Meetings.

1. María Cristina Escudero, Universidad de Chile, Chile. mcristina.escudero@iap.uchile.cl https://orcid.org/0000-0002-4908-3500 


\section{MECANISMOS CONSTITUYENTES Y EL CASO DE CHILE}

Existen distintos mecanismos para reemplazar una Constitución. La literatura ha clasificado estas instancias en tres: Comisiones Constituyentes, Congresos Constituyentes y Asambleas Constituyentes. Las comisiones constituyentes son órganos designados, por regla general, por el Ejecutivo, quien le hace el encargo de escribir la Constitución. En Latinoamérica, las comisiones han sido más comunes durante regímenes no democráticos, mientras que las Asambleas Constituyentes se han alzado en la actualidad como órganos cuyos integrantes han sido electos por el pueblo con el propósito de redactar una nueva Constitución (Negretto, 2018, p. 13).

Los Congresos Constituyentes (CC), en cambio, son los órganos legislativos de un sistema político a los cuales se les encomienda hacer una nueva Constitución. Como se trata de un órgano ya constituido, este se rige generalmente por las reglas a las que les debe su existencia y forma de funcionamiento. Los CC pueden ser a su vez de dos tipos. Por un lado, puede ser un CC que se elige con el propósito de redactar una nueva Constitución y luego de cumplido este mandato sigue sus funciones legislativas. Por otro lado, puede ser un CC que fue electo como Congreso y que asume luego un rol constituyente (PNUD, 2015, p. 11). Las fuentes de ese poder pueden ser múltiples: una solicitud del Ejecutivo, un mandato de la ciudadanía hecho mediante plebiscito; o porque el propio Congreso se auto arroga dicho rol. Sólo en el primer caso la ciudadanía sabe, al elegir sus representantes, que está delegando la facultad de hacer una Constitución y, por lo tanto, en el momento de la elección tiene en consideración las propuestas de los candidatos.

No obstante, la claridad con la que se puede dividir a estas tres familias de mecanismos, en lo sustantivo éstos no revisten las mismas características entre un proceso constituyente y otro. Un análisis de distintas experiencias refleja que el órgano mismo de decisión se combina con otros factores, como por ejemplo, la intervención del Ejecutivo o la participación ciudadana a través de plebiscitos o incluso la intervención conjunta de dos de estos órganos o los tres (Ginsburg, 2009, p. 5). Sin embargo, también revisando sus niveles de representación o participación, un mismo tipo de mecanismo puede variar sustancialmente. En otras palabras, cualquiera de estos mecanismos puede ser participativo o representativo o puede carecer de estas características. Solo considerando las AC que han tenido lugar en Latinoamérica, podemos observar que ellas han variado respecto a los grados de participación ciudadana, proporcionalidad de la representación, y los procedimientos internos mediante los cuales se toman las decisiones (Escudero, 2021a; PNUD, 2015, pp. 41-51).

Un punto relevante es la idea del poder extraordinario que tendrían los mecanismos constituyentes. Para algunos autores, la instancia deliberativa constituyente corresponde a un momento diferente a aquel de la política corriente que se manifiesta en el día a día de los procesos de toma de decisiones. En este sentido Elster (1995) argumenta que la elaboración de una Constitución exige un razonamiento imparcial y con visión de futuro, motivado por concepciones de interés público de un más largo plazo. En otras palabras, idealmente los momentos constituyentes quedarían a salvo de los intereses particulares. Otros autores argumentan que en la práctica, la política constituyente no se diferencia demasiado de la política distributiva que caracteriza la política ordinaria y, por el contrario, la deliberación está marcada por los intereses de las estructuras subyacentes que existen al momento del proceso (Negretto, 2013).

La noción de poder originario es otro elemento que genera expectativas en un proceso constituyente. Este se refiere a que el poder político vuelve al pueblo para redefinir las normas fundamentales los regulan (Ackerman, 1991). En oposición al poder constituido, aquel que existe y está regulado, el poder originario, que está en las bases del poder constituyente, tiene la capacidad de deliberar dándose sus propias normas porque es la base del ejercicio soberano y, de esta manera, se encontraría sobre las instituciones del Estado (Sieyès, 2003). Esta es una noción que se manifiesta con fuerza en las Asambleas Constituyentes latinoamericanas donde ha primado la idea que es el mecanismo de la expresión soberana.

Este artículo argumenta que las características que tiene la Convención Constitucional en Chile es consecuente con lo que la literatura identifica en otros procesos constituyentes en la región y que se traduce en las expectativas ciudadanas cuando enfrentan o demandan un proceso constituyente. En Chile, estas expectativas fueron madurando a lo largo de los años. Se toma como referencia para el análisis de este proceso de maduración las elecciones presidenciales de 2013 y el proceso de participación ciudadana para elaborar las 
Bases Ciudadanas de una Nueva Constitución, proceso constituyente impulsado por Bachelet en el 2016. De esta manera, el artículo se divide en tres partes. En la primera, desarrollo algunos conceptos básicos sobre mecanismos constituyentes. En la segunda, se desarrollan aspectos generales de las Asambleas Constituyente latinoamericanas contemporáneas y la experiencia chilena a lo largo de su historia. En la tercera parte, se presenta la relevancia de la AC en Chile en el periodo 1990 2018. En este se desarrollan brevemente algunos antecedentes de cómo madura la idea de AC y luego se describen las expectativas ciudadanas reflejadas, principalmente, en los Encuentros Locales Autoconvocados impulsados por el gobierno de Bachelet en el 2016. El artículo concluye que la forma en como se ha quedado conformada la Convención Constitucional es legítima desde el punto de vista de la representación de esta instancia, lo que es un buen pie de inicio para que la ciudadanía confíe en el proceso, aun cuando no garantiza su éxito.

\section{LA EXPERIENCIA LATINOAMERICANA Y CHILENA}

Los últimos procesos constituyentes latinoamericanos después de iniciada la tercera ola democrática, han sido todos protagonizados por Asambleas Constituyentes. Partiendo por Colombia en 1991, seguidos por Argentina en 1994, Ecuador en 1998, Venezuela en 1999, nuevamente Ecuador en el 2008 y finalmente Bolivia en 2009. Todos ellos han tenido distintas características de participación y representación. Por ejemplo, Colombia y Bolivia tuvieron ACs representativas y participativas, pero la participación de la primera fue de distinta naturaleza. En este país hubo plebiscito de entrada, cabildos antes de comenzar el trabajo de la AC y no hubo plebiscito ratificatorio. En Bolivia no hubo plebiscito de entrada, el proceso de participación fue a través de encuentros entre los Constituyentes y la ciudadanía en distintas partes del territorio, y contó con un plebiscito ratificatorio. En Venezuela, en cambio, la AC no fue representativa, la participación fue desestructurada aunque valorada por las organizaciones sociales, y al igual que en Bolivia, tuvo un plebiscito ratificatorio (Escudero, 2021a). Respecto al poder originario de las respectivas ACs, todas con excepción de Argentina y Ecuador en 1998, quisieron hacer gala de éste, lo cual fue logrado de distinta forma y alcance por Colombia, Venezuela y Ecuador en el 2008.
Chile se sumaría a esta cadena de reemplazos constitucionales realizados por gobiernos electos con su propia versión de Asamblea Constituyente, la primera desde su independencia. En su historia republicana, Chile ha tenido tres Constituciones estables, ninguna de las cuales ha sido una AC: La Constitución de 1833 para consolidar el orden conservador después de la Batalla de Lircay; la Constitución de 1925 tras la crisis social y política del orden oligárquico plasmado en la mal llamada república parlamentaria; y la Constitución del 1980 ideada después del golpe militar en 1973.

La Constitución de 1833 fue concebida por la llamada Gran Convención elegida por el Congreso con gran influencia del Ejecutivo, compuesta por 36 constituyentes (de los cuales 30 eran parlamentarios). Con todo, el trabajo de redacción del borrador fue encargado a una comisión de siete personas y éste fue aprobado por aquella convención (Correa, 2015, p. 46). La Constitución de 1925 fue redactada también por una comisión. En los hechos, la intención del presidente Alessandri era llamar a una Asamblea Constituyente. Sin embargo, finalmente designó una comisión de 122 personas con representación de todos los sectores políticos existentes en la época. Finalmente, no fue esta instancia la que redactó la Constitución, sino que Alessandri creó una subcomisión de 15 miembros, presidida por él, que redactaron el proyecto de Constitución, el que fue aprobado en plebiscito (Faundez, 2011, p. 84). Por último, la Constitución de 1980 fue encargada por la Junta Militar a una comisión de estudios, cuyo borrador fue revisado por el Consejo de Estado (designado por la Junta) y el proyecto final escrito por una nueva comisión. El texto fue sometido a plebiscito, que sin contar con las garantías mínimas de transparencia, lo aprobó (Correa, 2015, pp. 66-67).

La nueva Constitución que reemplace la Constitución de 1980 será redactada por una Convención Constitucional que en esencia es una Asamblea Constituyente. El hecho que la ciudadanía se inclinara por esta opción es consecuente con lo que los movimientos sociales y las encuestas de opinión pública venían mostrando los años anteriores. Sin embargo, en la discusión pública nunca se profundizaron las características o las expectativas que sus partidarios depositaban en este tipo de mecanismo, más allá del hecho de ser una instancia distinta del Congreso.

Las elecciones para elegir a los miembros de la Convención Constitucional se realizaron el 15 y 16 de mayo de 2021. En esta elección la ciudadanía 
tuvo preferencias de candidatos muy distintas a las que se reflejan en el Congreso elegido en el 2017. En primer lugar, optó por candidaturas independientes incluso al interior de la lista de partidos. En segundo lugar, se premió un perfil ciudadano con trayectoria en los territorios o movimientos sociales o por independientes profesionales e intelectuales progresistas. Tercero, la composición ideológica de los convencionales constituyentes tiene una marcada inclinación hacia la izquierda. De este modo, la Convención, de 155 miembros, quedó conformada por 37 convencionales de la lista de partidos de centro-derecha; 25 convencionales para la lista de partidos tradicionales de centro-izquierda; 28 convencionales para los partidos de izquierda no tradicional; 27 convencionales para independientes de la Lista del Pueblo; 11 convencionales para la Lista de Independientes no Neutrales y 10 independientes. Adicionalmente, los pueblos originarios se eligieron 17 convencionales (gráfico $n^{\circ} .1$ ).

\section{Gráfico 1}

Resultados de elección de Convencionales Constituyentes 2021

\section{Convencionales Constituyentes por Lista}

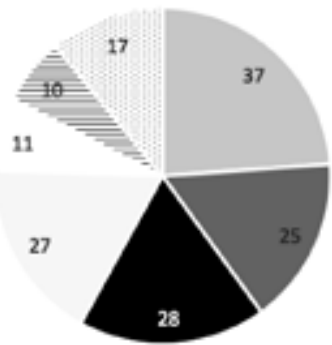

"Centro - Derecha

- Centro - Irquierda Tradicional

- isquierda

Ind. Usta del Pueblo

Independientes No Neutrales

= Otros Independientes

Pueblos indigenas

Fuente: Elaboración propia a partir de datos obtenidos en www.servel.cl

La Convención también es paritaria ya que fue elegida con un sistema electoral que permitió en los resultados contar con igual número de hombres que de mujeres ( 78 hombres y 77 mujeres), lo que no solo es extraordinario en Chile, sino que también es la primera experiencia constituyente en el mundo con esta característica. A esto se suma su carácter pluricultural o nacional, al incluir a más del 10\% de pueblos indígenas. En resumen, es de mucha diversidad social, es paritaria y tiene representación de los pueblos originarios. La Convención se convirtió en un reflejo mucho más cercano de lo que es la sociedad actual, de lo que se proyectaba antes de la elección.

\section{CHILE ANTE LA ASAMBLEA CONSTITUYENTE: 1990 - 2018}

\subsection{Antecedentes}

Hasta el acuerdo político "Por la Paz y la Nueva Constitución" el 15 de noviembre de 2019, la Constitución de 1980 había tenido la capacidad de sobrevivir a su reemplazo. Sin embargo, en distintos momentos a partir de su promulgación, han existido sectores, tanto políticos como sociales, que han empujado una nueva Constitución y una Asamblea Constituyente (AC) que plasme ese anhelo. Así, antes de su promulgación, la oposición a la dictadura de Pinochet (1973-1990) Ilamaba a oponerse a ella. Un mes antes del plebiscito de 1980, que sin garantías mínimas de transparencia aprobó el texto constitucional, el ex presidente Frei Montalva llamaba a su rechazo señalando: "Todos su contenido revela una confianza ilimitada en los mecanismos de concentración de poder, y una desconfianza igualmente ilimitada en el pueblo, en el Parlamento y en los partidos políticos, en los organismos de base y hasta en los municipios" (Frei Montalva, 1980). ${ }^{1}$ Para Frei Montalva, la faltas de garantías del plebiscito de 1980 en plena dictadura militar, hacían inevitable la aprobación de la constitución, sin perjuicio de lo cual, no dudó en recalcar que los caminos a la libertad debían hacerse por un gobierno provisional que convocara a una $\mathrm{AC} u$ otro órgano ampliamente representativo.

Una vez inaugurada la democracia, la Concertación -la alianza de centro izquierda que gobernó el país desde 1990 al 2010- optó por buscar reformas a la Constitución utilizando su marco jurídico. Al frente se encontró con una decidida oposición de derecha y militar fortalecida por los 'enclaves autoritarios' que la misma Constitución establecía, que no permitió cambios sustantivos a ella, sino hasta el 2005, quince años después (Garretón, 2003; Fuentes, 2012). ${ }^{2}$ Durante este tiempo, hubo sectores en la iz-

1. https://www.casamuseoeduardofrei.cl/wp-content/ uploads/2009/04/Discurso-con-motivo-del-Plebiscito-de-1980.pdf

2. Los 'enclaves autoritarios' son mecanismos institucionales que hicieron posible perpetuar el legado de la dictadura después de hecha la transición a la democracia y que dieron protagonismo político a las FF.AA y que han dificultado la expresión de la mayorías en el proceso de toma de decisiones favoreciendo la posición política de la derecha, como por ejemplo, la existencia de senadores designados, el sistemas electoral binominal y altos quorums legislativos (Garretón, 2003). 
quierda extraparlamentaria que siguieron insistiendo en el reemplazo de la Constitución por la vía de una Asamblea Constituyente. Todas las plataformas de campañas presidenciales de este sector, recogían la propuesta de una nueva Constitución concebida por una AC (Marin, 1999; Hirsh, 2005). A partir del 2009, la Concertación que gobernó el país hasta el 2010 y posteriormente la Nueva Mayoría -la continuación de la Concertación más el Partido Comunista- incorporaron la propuesta de una nueva Constitución, pero sin definición clara del mecanismo constituyente para llevarla a adelante. En el caso de la fallida campaña presidencial de Frei Ruiz-Tagle en el 2005, la propuesta de programa de gobierno hacía referencia a una comisión representativa que hiciera un borrador y una propuesta de mecanismo para la aprobación de la Constitución del Bicentenario (Frei, 2009), mientras que Bachelet en el 2013 propuso una nueva Constitución hecha de una manera "institucional, democrática y participativa” (Bachelet, 2013).

En los hechos, la idea de una AC es puesta en el debate público por la ciudadanía. Lentamente la $\mathrm{AC}$ se apodera del debate a través de los movimientos sociales, especialmente aquellos que llenaron las calles a partir del 2011 y el movimiento Marca tu Voto o Marca AC que levantó esta bandera para las elecciones presidenciales del 2013 y jugó un rol relevante incentivando la discusión y el debate político en tono a la $\mathrm{AC}$, al promover que la gente marcara su voto con una $\mathrm{AC}$ al momento de acudir a las urnas (Escudero, 2021b). En el contexto de la elección presidencial de 2013, siete de los nueve candidatos que se presentaron en primera vuelta propusieron una nueva Constitución y de ellos 5 se inclinaron por una $\mathrm{AC}$ aunque ninguno de ellos elaboró una propuesta concreta sobre las características de este mecanismo.

En paralelo a la discusión política, comienzan a visibilizase en la ciudadanía organizaciones sociales pro AC en los espacios académicos alternativos y en las redes sociales. Por ejemplo, se activa el Movimiento por una Asamblea Constituyente y se impulsan "Escuelas por la Asamblea Constituyente". En torno a estas iniciativas y el movimiento estudiantil, hay un esfuerzo adicional por explicar lo que implica una $\mathrm{AC}$ y un proceso constituyente en términos más generales. En la mayoría de los casos, la AC se explica como una elección ciudadana de delegados con el único objetivo de elaborar una Constitución amparada en la noción de que es el pueblo el único depositario de la soberanía nacional, con poderes por sobre aquellos establecidos (Grez, 2015). Este debate no tuvo eco en los medios de comunicación.
Tanto en la esfera política como en los movimientos sociales, se manejan definiciones generales de lo que debe ser una AC. Para los actores políticos, la clave es la elección de representantes en una instancia de debate constitucional. Esta elección se entiende similar a la conocida para el Congreso donde los partidos políticos son protagonistas de ella. Para los movimientos sociales es especialmente relevante que sea la ciudadanía la que decida "desde abajo" y que se rompa la tradición chilena de tomar decisiones constituyentes solo desde la elite (Salazar, 2015) Más aún, la AC es planteada como el único medio realmente democrático fundamentado en la participación de personas distintas a las elites tradicionales. A grandes rasgos estas parecen ser las expectativas que la ciudadanía tenía respecto de una $\mathrm{AC}$ al comenzar el gobierno de Bachelet en el 2014, sin embargo, el debate político no logró concretar ningún mecanismo constituyente que sirviera de vehículo al reemplazo constitucional.

\subsection{Encuentros Locales Autoconvoca- dos y Cabildos 2016: La AC se posiciona}

Al asumir Bachelet su segundo gobierno en el 2014, comenzó a implementar su compromiso de campaña de realizar el reemplazo de la Constitución mediante un proceso institucional, democrático y participativo. Los desacuerdos en el mecanismo constituyente entre y al interior de los partidos de la Nueva Mayoría -la coalición de centro-izquierda que llevó a Bachelet a la presidencia- retrasó esta decisión e hizo optar al equipo encargado del proceso constituyente. Esto hizo que el gobierno se decidiera por comenzar un proceso de participación ciudadana que le permitiera elaborar las "Bases Ciudadanas para la Nueva Constitución" mientras se buscaban los acuerdos en torno al órgano responsable del reemplazo (Escudero, 2021c).

El proceso de participación ciudadana en 2016 tuvo 4 etapas (Consulta Individual, Encuentros Locales Autoconvocados o ELAs; Cabildos Provinciales y Cabildos Regionales). En total participaron más de 200 mil personas, constituyéndose en el proceso de participación con objetivos constituyentes con más convocatoria en Latinoamérica después de la tercera ola democrática, medido porcentualmente sobre la población total (Informe OCDE, 2017, p. 24; Escudero, 2021a). En términos generales, esta instancia permitió a sus participantes pronunciarse sobre las prioridades que tenían respecto a los principios y valores, derechos, obligaciones e insti- 
tuciones que debían ser incluidos en la nueva Constitución, y sus fundamentos.

Todas las instancias de este proceso participativo fueron sistematizadas, por lo que es posible conocer la prioridad que los participantes le daban en ese momento a la AC y los fundamentos de su relevancia. En este contexto, el movimiento Marca $A C$ que se había logrado organizar para las elecciones del 2013, utilizó la red que había creado con el propósito de relevar la importancia de la AC en los ELAs y los Cabildos (Escudero, 2021c). En suma, hizo una campaña para que los participantes incluyeran la AC como una prioridad en la Constitución y de esta manera presionar para que se convirtiera en el mecanismo constituyente. La estrategia fue exitosa, toda vez que la AC logra un lugar destacado en la prioridad que le dan los participantes en su deliberación, pese a los obstáculos para que así fuere. En efecto, la forma como se posiciona la AC en este proceso participativo, debió sortear la ausencia expresa de una referencia a ella en los documentos metodológicos que servían de base a los participantes para la discusión.

La metodología juega un rol importante a la hora de entender el posicionamiento ciudadano respecto de la AC. Cuando se decidieron los instrumentos que se entregarían a los participantes para incentivar la discusión al interior de los ELAs o cabildos, el Consejo de Observadores Ciudadanos (CCO) no estuvo de acuerdo en incluir la Asamblea Constituyente como una alternativa explicita en los documentos de deliberación ${ }^{3}$. Es decir, el instrumento que orientó la discusión sobre instituciones, proponía priorizar 7 de ellas de una lista de 22 instituciones donde no figuraba la opción AC. En su lugar, incluyó una cate- goría para "otros" donde la ciudadanía podía expresar libremente otras preferencias. Por lo tanto, un tema en particular que no estuviese incluido en los documentos que guiaban la discusión de los participantes podía ser propuesto, pero para llegar a destacar en los resultados, se requería de una buena capacidad de organización y movilización que congregara muchas menciones. Ello para sobrepasar la ventaja con la que partían las instituciones que sí tenían una mención expresa en el instrumento que la ciudadanía tenía a disposición para deliberar.

La campaña de Marca AC fue exitosa. En los ELAs la Asamblea Constituyente alcanzó el lugar 18 entre las instituciones mencionadas como deseables de incluir en la Constitución. Es decir, superó a algunas instituciones que explícitamente se encontraban en el documento y logró concentrar preferencias en la categoría de "otros". La inclusión de la AC en el debate del proceso participativo del 2016 se intensificó en las etapas siguientes. En los cabildos provinciales, la Asamblea Constituyente escaló hasta el séptimo lugar. Esta tendencia es especialmente fuerte en los cabildos provinciales hechos en la región de Los Lagos ( $6^{\circ}$ lugar) y en la región de los Ríos (3er lugar), mientras que la RM, lo coloca en el $10^{\circ}$ lugar. En la etapa siguiente -Cabildos Regionales-, la AC ocupa el quinto lugar de las instituciones priorizadas. Nuevamente son los cabildos de las regiones fuera de Santiago los que manifiestan con mayor fuerza esta opción: Antofagasta, Valparaíso y Los Ríos, seguidas por Los Lagos y Aisén (Documentos de sistematización, 2017, p. s/n).

El éxito de esta estrategia de inclusión de la AC en las "Bases Ciudadanas para una Nueva Constitución”, se alineaba con la opinión pública. En la

Tabla 1. Mecanismo Constituyente

\begin{tabular}{|l|c|}
\hline Tipo de mecanismo seleccionado & $\%$ \\
\hline Grupo de expertos que redacte una Nueva Constitución & 22 \\
\hline Parlamento que redacte una nueva Constitución & 4 \\
\hline Asamblea Constituyente elegida por la ciudadanía para redactar una Nueva Constitución & 65 \\
\hline NS/NR & 8 \\
\hline
\end{tabular}

Fuente: Encuesta COES 2016. Porcentajes no suman 100\% por aproximación decimal de los resultados

3. El CCO fue un órgano nombrado por la Presidenta Bachelet para hacer recomendaciones al proceso y dar garantías de imparcialidad al mismo. Dado su rol este fue compuesto por representantes de todos los sectores políticos y algunos representantes no partidarios. 
época en que se realizaban los ELAs y Cabildos, la encuesta COES 2016 mostraba una inclinación mayoritaria (56\%) que consideraba importante remplazar la Constitución de 1980 frente a un 13\% que no consideraba importante hacerlo. Estos resultados se corresponden con el $55 \%$ que se manifestaba disconforme con la actual Constitución. Teniendo esto como contexto, cuando se les consulta a las personas por el mecanismo que prefieren para reemplazar la Constitución, un $65 \%$ se manifiesta a favor que sea una AC la encargada de hacerlo, frente a un $22 \%$ que prefiere una comisión de expertos y un $4 \%$ que menciona el Congreso.

Los resultados muestran al Congreso como una posibilidad constituyente disminuida, incluso frente a la comisión de expertos. En el fondo, la opción de una $\mathrm{AC}$ no tiene contrapeso en la opinión pública. El proceso participativo de 2016 tiene la virtud de ayudarnos a comprender de mejor manera qué esperaba la gente respecto de una eventual Asamblea Constituyente.

\subsection{Las aspiraciones depositadas en una AC}

Parte del ejercicio de participación de 2016 consistió en fundamentar las opciones tomadas por los participantes. Los fundamentos también fueron sistematizados por un comité independiente y entregados como parte de las bases ciudadanas (Informe OCDE, 2017). Los principales productos de esta sistematización fueron ideas fuerzas que representaban la tendencia general relacionada con aspectos descriptivos, valorativos y propositivos (Comité de Sistematización, 2017, p. 11).

Respecto de la AC, los fundamentos expresan características bastante específicas respecto de ella. La idea fuerza expresada en los documentos de sistematización del proceso participativo, relevó la idea que este mecanismo es visto como el medio más democrático y representativo para crear una Constitución. A esto se suma que la AC se entiende como el espacio donde se les da a los ciudadanos capacidad de participar y decidir en forma incidente (e incluso vinculante) de forma diferenciada a la hegemonía de los partidos. En suma, se distingue a la AC como una instancia ciudadana (Documentos de Sistematización, 2017, p. s/n).

En forma similar a los ELAs, los cabildos provinciales, caracterizaron la AC como una "forma única de participación ciudadana", que "otorga legitimidad al cambio constitucional". Se entiende, también, como un procedimiento incidente haciéndolo sinónimo de vinculante. En consecuencia, el concepto que se tiene de $\mathrm{AC}$ también se relaciona con la imagen de un mecanismo democrático para reemplazar la Constitución donde el rol de la gente común es fundamental. La fundamentación en los cabildos regionales es mucho más excluyente respecto de otros mecanismos constituyentes, aunque apunta en la misma dirección. En efecto, se percibe la AC como "el único mecanismo o forma de control y de participación legítima asociada a una nueva Constitución" donde también se le da el carácter vinculante y se repite la noción de "popular", probablemente relevando la idea de una composición más ciudadana y no dominada por los partidos políticos como es en el Congreso (Documentos de Sistematización, 2017, p. s/n).

Un nuevo análisis de los resultados de los ELAs a partir de las bases de datos puestas a disposición por el Comité de Sistematización, permiten profundizar en las expectativas ciudadanas respecto a la AC. Para este nuevo análisis se realizó un ejercicio deductivo para identificar ideas centrales o énfasis que los participantes quisieron comunicar, buscando profundizar respecto de las ideas fuerzas que

\section{Tabla 2}

Ideas centrales o énfasis expresado en fundamentos de los ELA que señalaron AC

\begin{tabular}{|c|l|c|}
\hline & Ideas Centrales o énfasis & \\
\hline 1 & Mecanismo soberano de participación y decisión & 238 \\
\hline 2 & Mecanismo con una finalidad constituyente específica & 169 \\
\hline 3 & El único o el mejor mecanismo constituyente & 140 \\
\hline 4 & Institución que potencia ejercicio democrático & 92 \\
\hline 5 & Mecanismo que debe institucionalizarse & 87 \\
\hline 0 & No menciona fundamentación (residual) & 80 \\
\hline Total 1 a 5 & & 726 \\
\hline Total & & 806 \\
\hline
\end{tabular}

Fuente: Elaboración propia a partir de los fundamentos de ELAs 
se produjeron en la sistematización del proceso, aprovechando la posibilidad de aplicar otras técnicas interpretativas.

En la base de datos se identificaron 806 ELAs que priorizaron la AC como una institución a ser considerada en la nueva Constitución. De éstas, 726 fundamentaron su respuesta. Al analizarlas, fue posible agruparlas en cinco familias de ideas centrales o énfasis (Tabla 2): a) Un 32,8\% (238 casos) ven la AC como un mecanismo soberano de participación y decisión; b) Un 23,3\% describen el mecanismo con una finalidad constituyente específica; c) Un 19,3\% consideran la AC como el único o mejor mecanismo constituyente; d) Un $12,7 \%$ entiende la AC como una institución que potencia el ejercicio democrático; y d) un 12\% pone énfasis en la necesidad de institucionalizarla. Por último, hay 80 casos, un 11\%, que no fundamentaron su elección.

Como mecanismo soberano de participación y decisión resalta el énfasis de la AC como el lugar donde radica el poder político, vinculado a la idea que el poder constituyente reside en la ciudadanía o el pueblo, quienes tienen el derecho de darse una Constitución. En otras palabras, el centro de esta categoría es que en la AC radica el poder de decisión, ahí se decide. Aunque no siempre se señala explícitamente la finalidad del mecanismo, su función principal descansará sobre acciones más resolutivas como generar, decidir y crear una nueva Constitución. Lo más importante para los participantes de estos ELAs es la integración del mecanismo y su naturaleza. Junto con fundamentarla como mecanismo soberano, la idea de mecanismo ciudadano o popular guía las expectativas de estos fundamentos. Frases como "la ciudadanía debe ejercer la soberanía para reformar o cambiar la misma" o "Por el reconocimiento constitucional a la soberanía del pueblo y su derecho a generar nuevas constituciones futuras" dan cuentan de la intensidad con la que se busca un mecanismo donde radique el poder constituyente directo, es decir, sin los intermediarios institucionalizados.

Las expresiones señaladas, revindican a los integrantes de la sociedad como tales, ya sea el pueblo, ya sea la ciudadanía, desde donde el resto de los poderes emana. Muchas fundamentaciones utilizan la expresión concreta de 'soberanía', otras lo señalan implícitamente al verse como conformando una institución diferente a la del Congreso y otra forma de cambio constitucional. En este sentido, expresiones que dan cuenta de la necesidad de “... poder arrogar al pueblo el derecho de poder construir una nueva carta magna" excluye a un "otros" que no son el pueblo y que ejercen o se arrogan dicho poder. De esta manera, el hecho que "Chile necesita un espacio de representación real del pueblo. Suplantar el Congreso Nacional actual por un espacio democrático y resolutivo" es parte de ese anhelo de que la soberanía se recrea en una $A C$, no en el Congreso.

En la idea de mecanismo soberano de participación y decisión, descansa también en las expresiones sobre su naturaleza. Como es de esperarse, la propiedad más mencionada es que la naturaleza propia de la AC es ser participativa, pero también inclusiva, es decir, que acoja a la diversidad. Hay un conjunto de fundamentaciones que enfatiza la importancia de un todos: "que todos y todas participemos en igualdad de condiciones", "En la que todas las personas participen", pero que también recoja la pluralidad... "integrando la participación de los distintos actores que conforman nuestra comunidad".

La AC como mecanismo soberano también descansa sobre un cierto grado de superioridad frente a otras alternativas. Los fundamentos hablan de que "esta institución debe ser entendida como un espacio de real participación democrática..." o es donde se da "el ejercicio más profundo y democrático de soberanía que puede darse un pueblo...". Por último, esta es la única idea central que destaca por referirse también al carácter originario de la AC. Detrás de esta propiedad se describe a una AC como depositaria del poder constituyente originario "sin tener más límites que los que la propia voluntad popular determine", poniéndola por encima de todas las otras formas de decisión política.

En segundo lugar, se describe la AC centrada en su finalidad. En otras palabras, los participantes mediante la fundamentación enfatizan lo que imaginan lo que la AC debiera hacer o para lo que sirve. Esta categoría se funde con el subgrupo que identifica la acción esperada por la AC, ya que quienes fundamentan la AC poniendo énfasis en su finalidad lo hacen a través de la acción principal que realizará. Para quienes enfatizan la finalidad, la idea central es que la AC sea la encargada de redactar la nueva Constitución. Así, para la mayoría de los ELAs en esta categoría, la AC “...es el mecanismo que debiese ocuparse para la redacción de la Constitución..." o como órgano "encargado de la redacción de una nueva Constitución". Además de la redacción asoma como importante, que la AC sea la encargada de cambiar la Constitución, sin especificar si este será un cambio total 
o parcial, lo que sí hace cuando se refiere a otras acciones que implican el cambio total como crear o generar una nueva Constitución. Estas tres finalidades ocupan aproximadamente dos tercios de la idea principal de lo que haría una AC.

Esta forma de fundamentar la AC también está marcada por la importancia que se le da a la naturaleza del mecanismo. En este sentido, la virtud que se le asigna a la AC es que sea electa popularmente de tal manera que se transforme en la "Institución compuesta por ciudadanos no incumbentes elegidos por la ciudadanía, cuya única función es redactar la constitución", pero también con la característica de ser participativa y de carácter permanente. Así, constituir una AC “Para redactar una nueva constitución con amplia participación ciudadana" es la expectativa de quienes abogan por este tipo de mecanismos, a lo que agregan su institucionalización: “... debe ser permanente y ser convocada en cada situación que lo amerite". Llama la atención, que para este grupo, el carácter ciudadano y popular es tan importante como otras expresiones: representantes, asambleístas o categorías individuales como personas o gente, que no tienen una connotación cívica o de pertenencia tan fuerte.

El tercer grupo identificado en los fundamentos es aquel que considera la $\mathrm{AC}$ como un mecanismo constituyente superior a otras alternativas. En consecuencia, la AC se trata de el mejor o el único mecanismo. Es aquel que tiene un carácter realmente constituyente vis à vis otros. A éste se le valora preferentemente por su naturaleza y no tanto por su función o integración. En los hechos, se le consigna su superioridad democrática y como instancia legítima para albergar el poder constituyente. Más de la mitad de las menciones sobre la naturaleza de esta instancia única o mejor, hace referencia a estas dos características, de tal manera que la $\mathrm{AC}$ es vista "Como institución única para modificar democráticamente la constitución y promover así el bien común" o bien se le justifica porque "Es fundamental que se establezca un mecanismo de la máxima legitimidad para nuestra carta fundamental" o la forma "más democrática y con garantías de participación real de la ciudadanía".

El carácter de la AC también tiene efectos que van más allá solo de su superioridad respecto a otros mecanismos. Uno de cada cuatro ELAs que enfatizan la naturaleza de la AC en este grupo, vaIora su ocurrencia porque tiene un impacto en la nueva Constitución, en el proceso o en el sistema político. La AC tendría la virtud de validar o legiti- mar la Constitución que emane de ella, para muchos es el "requisito básico para una nueva Constitución democrática" o la única forma de legitimar una Constitución. Es, como señala uno de los fundamentos, la "institución fundamental para que la nueva constitución sea válida".

Su existencia también garantizaría una democratización del sistema político y con ello también una legitimización del mismo. De alguna manera, la AC por sí misma, podría resolver los problemas de confianza que las personas ven con la institucionalidad vigente. A este respecto, la excepcionalidad de la AC se daría porque tendría la virtud de "reconstruir la legitimidad del sistema político..." y "mejorar las condiciones del estado y su relación con los ciudadanos".

La función más relevante para quienes ponen énfasis en la excepcionalidad de la AC, es la acción que trae como consecuencia el reemplazo de la Constitución, como construir una nueva constitución o crearla. En los hechos, los ELAs de este grupo hablan de una institución "fundamental para construir una Constitución verdaderamente legítima". La integración del mecanismo pierde relevancia en este grupo, aunque aquellas fundamentaciones que la mencionan, se inclinan mayoritariamente por una integración ciudadana y en menor medida, del pueblo.

La cuarta idea central que destaca en el análisis de los ELAs es aquella que enfatiza las características del proceso de toma de decisiones que prevalece en la AC. De esta manera, se pone mucho acento en su naturaleza participativa y en la toma de decisiones democrática. En los hechos, la expectativa más grande que se expresa en este tipo de fundamentación, es la posibilidad de participar del proceso. El objetivo no es el resultado de la nueva Constitución, sino que es vista como un "mecanismo para participar democráticamente". Se utilizan expresiones como "un mecanismo para dar una voz válida a la ciudadanía" o para "resolver pacíficamente desencuentros institucionales". En este contexto, se trata de fundamentaciones que no hacen una mayor especificación de la acción que pretende, ni tampoco de su integración, aunque en el primer caso se centra en acciones intermedias, como discutir o debatir y, en el segundo caso, en su integración ciudadana.

Por último, hay un grupo de ELAs que enfatiza la necesidad de que éste se institucionalice, principalmente explicitando la necesidad que se incluya en la Constitución. Esto se justifica generalmente con la naturaleza permanente que se le otorga, 
regulado para que sea utilizado cuando sea necesario, en otras palabras, de acuerdo a esta categoría, "debe existir una institución permanente que se conforme en cada reforma constitucional" o al menos cuando se discuta una nueva Constitución. Por otra parte, más de la mitad de las funciones que se le asignan a la AC, son para cambiarla (sin referencia a si es un cambio total o parcial) o modificarla, como se ilustra en la frase recién citada. En consecuencia, con esta fundamentación se buscar tener certeza que existirá en el futuro y que el pueblo (y la ciudadanía en menor medida) contará con ella. Para otros dentro de esta categoría, el carácter permanente se expresa en la posibilidad que la AC se pueda autoconvocar.

\section{CONCLUSIONES}

Las demandas por una Asamblea Constituyente en Chile comienzan desde las movilizaciones sociales que se toman las calles a partir del 2006 y se acentúan con la segunda ola de protestas en el 2011. En la antesala de las elecciones del 2013 existieron movilizaciones sociales que con fuerza empujaron la idea de tener una AC. Esto impactó a nivel político y se convirtió en debate obligado entre las distintas candidaturas. Sin embargo, la elite política no logró un consenso en torno a la posibilidad de tener una $\mathrm{AC}$ y en los hechos la resistencia para una AC es desbordada por las organizaciones sociales, que le dan visibilidad.

Para las organizaciones sociales de la época, una $\mathrm{AC}$ representaba principalmente la única posibilidad democrática y participativa que refleja la soberanía del pueblo. Esto se condice con los resultados entregados por el Comité de Sistematización de la etapa participativa para la elaboración de las Bases Ciudadanas para una Nueva Constitución de 2016, en los que sobresalen como ideas fuerzas, conceptos similares. La AC sería el mecanismo más democrático, representativo y participativo. La AC es valorada como instancia ciudadana y legítima que prescinde de poderes constituidos. También se reporta la expectativa de que sea vinculante.

Un nuevo análisis de la base de datos complementa las conclusiones anteriores. Así, cuando se habla de la AC como mecanismo soberano de participación y decisión, se enfatizan ideas relacionadas con su integración popular o ciudadana, donde la participación se da en forma inclusiva y diversa.
De esta manera, una AC tendría una naturaleza más democrática o legítima para involucrarse en los temas constituyentes donde explícitamente se le caracteriza como originaria. Adicionalmente, es un mecanismo con una finalidad constituyente específica, es decir, la AC existe para redactar una nueva Constitución, lo que debe hacerse de forma participativa. No hay fuerza en la idea que el poder originario puede intervenir otros poderes del Estado o tomar decisiones que no estén en el marco de darle al país una nueva constitución.

La AC es pensada en su carácter extraordinario o en otras palabras, como único o el mejor mecanismo constituyente, que es excluyente frente a otras alternativas dadas sus características excepcionales: democrática y legítima. La excepcionalidad también se explica por su carácter democratizador y legitimador del proceso, la Constitución y el sistema político. Para algunos, esto amerita que la $\mathrm{AC}$ se institucionalice e incluso tenga un carácter permanente. En resumen, las expectativas frente a una $\mathrm{AC}$ son altas.

Del análisis se desprende que lo que la ciudadanía esperaba en Chile no están lejos de las aspiraciones de otros constituyentes latinoamericanas recientes. Más aún, no sólo existe un correlato entre lo que manifestaron los participantes del proceso participativo de 2016 y otras experiencias de la región, sino que estas expectativas confirman lo que parte de la literatura reflexiona en torno al poder originario y el poder constituyente.

Los resultados de la Convención Constitucional el 15 y 16 de mayo de 2021 son consecuentes también con lo que se esperaba para una instancia como esta. Finalmente, la ciudadanía fue superando uno a uno los obstáculos para tener un proceso constituyente a través de una AC que cumpliera con las características anteriores. Aunque no sabemos aún si la $A C$ tendrá éxito en su tarea, sí es posible constatar que el fuerte componente de independientes que le han restado protagonismo a los partidos y la diversidad de la representación que alcanzó, la han dotado de un semblante de legitimidad que no habría obtenido de otra manera a la luz de los datos que se han presentado. Este es un buen capital de inicio para la deliberación que debe librar la Convención y, aunque no es todo lo que se requiere para su éxito, sí es un factor relevante para comprender el desarrollo del proceso constituyente chileno. 


\section{REFERENCIAS}

Ackerman, B. (1991). We the People, Volume I: Foundations. Harvard University Press.

Bachelet, M. (2013). Programa de gobierno (20142018). Biblioteca Nacional Digital. http://www. bibliotecanacionaldigital.gob.cl/bnd/646/ w3-article-157782.html

Bases de Datos Sistematización (2017). Archivo de la Biblioteca Nacional.

COES (2016). ELSOC Survey Team (2018): "Estudio Longitudinal Social de Chile 2016", https://doi. org/10.7910/DVN/OKIRBJ, Harvard Dataverse, V2, UNF:6:r/EWvQBdjHYNE7dU//d1VA== [fileUNF]

Comité de Sistematización (2017). Informe Ejecutivo. Archivo de la Biblioteca Nacional

Correa, S. (2015). Los procesos constituyentes en la historia de chile. Estudios Públicos (137), pp. 43-85

Documentos de Sistematización (2017). Archivo de la Biblioteca Nacional

ELSTER, J. (1995). Forces and Mechanisms of the Constitution-Making Process. Duke Law Journal (45), pp. 364-396. https://doi.org/10.2307/1372906

Escudero, M. C. (2021a). Asambleas Constituyentes en Latinoamérica. Aspiraciones de Igualdad, Participación y Libertad. Ediciones LOM.

Escudero, M. C. (2021b). Momento Constituyente. En J. Gajardo (Ed.). La ruptura constituyente. Rubicón Editores (en prensa).

Escudero, M. C. (2021c). Making a Constituent Assembly Possible in Chile: The Shifting Costs of Opposing Change. Bulletin for Latin American Research, 1-16. https://doi.org/10.1002/ BLAR.13290

FAúnDEZ, J. (2011). Democratización, Desarrollo y Legalidad: Chile 1831 - 1973. Ediciones Universidad Diego Portales

FREI, E. (2009, 8 de septiembre). Discurso entrega de programa 7 de septiembre de 2009. https:// es.slideshare.net/equipofrei/discurso-de-eduardo-frei-ruiztagle

Fuentes, C. (2012). El pacto. Poder, constitución y prácticas políticas en Chile (1990 - 2020). Ediciones Universidad Diego Portales.

Garretón, M. A. (2003). Incomplete Democracy. University of North Carolina Press

Ginsburg, T., Z. Elkins y J. Blount (2009). Does the Process of Constitutional Making Matters?. The Annual Review of Law and Social Science, (5), 201-23. https://doi.org/10.1146/annurev.lawsocsci.4.110707.172247
Grez, S. (2015). Asamblea Constituyente. La alternativa democrática para Chile. Editorial América en Movimiento.

HiRsch, T. (2005). Plataforma Programática para un Chile Justo, Solidario, Soberano y Democrático. EMOL. https://www.emol.com/especiales/_elecciones_2005/descargas/programa_Tomas_Hirsch.pdf

INFORME OCDE (2017). Chile, La Participación Ciudadana en el Proceso Constituyente 2017

MARIN, G. (1999). Programa candidatura presidencial de Gladys Marín. Archivo Chile. http://www.archivochile.com/Izquierda_chilena/frentes_polit/ otros_doc_ich/ICHotrosdoc0007.pdf

Negretto, G. (2013). Making Constitutions: Presidents, Parties and Institutional Choice in Latin America. Cambridge University Press. https:// doi.org/10.1017/CB09781139207836

Negretto, G. (2018). Constitution-Building Processes in Latin America. International IDEA Discussion Paper. https://doi.org/10.31752/idea.2018.10

PNUD (2015). Mecanismos de cambio constitucional en el mundo. Análisis desde la experiencia comparada. PNUD

SAlAZAR, G. (2015, 15 de diciembre). Asamblea constituyente: ¿chile está preparado? Asamblea Constituyente. http://www.asambleaconstituyente. cl/2015/12/asamblea-constituyente-chile-esta. html

SIEYĖs, E. (2003). Political Writings. Hackett Publishing. 